\title{
Correction of Adolescent Blount's Disease Deformity Using Hinged Ilizarov Frame
}

ISSN: 2576-8875

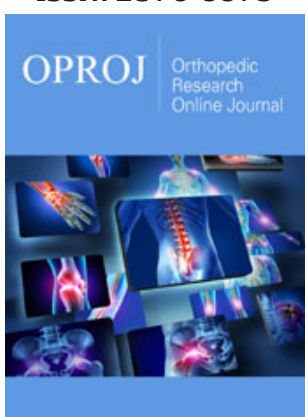

*Corresponding author: Mustafa Abd El Mogeeb Radwan, Department of Orthopedic, Mansoura Health Insurance Hospital, Mansoura, Egypt

Submission: 誨 January 29, 2020

Published: 傮 February 04, 2020

Volume 6 - Issue 4

How to cite this article: Mustafa Abd E Mogeeb Radwan. Correction of Adolescent Blount's Disease Deformity Using Hinged Ilizarov Frame. Ortho Res Online J. 6(4). OPROJ.000643.2020.

DOI: 10.31031/OPROJ.2020.06.000643

Copyright@: Mustafa Abd El Mogeeb Radwan, This article is distributed under the terms of the Creative Commons Attribution 4.0 International License, which permits unrestricted use and redistribution provided that the original author and source are credited.

\author{
Mustafa Abd El Mogeeb Radwan* \\ Department of Orthopedic, Egypt
}

\begin{abstract}
Background: Adolescent Blount disease is the commonest cause of genu varum in old children. It is a multiplanar deformity consisting of varus, procurvatum and internal tibial torsion. Ilizarov frame can be used for both acute and gradual correction. Acute correction has the benefit of shorter time to healing and more comfortable to the patient. Gradual correction needs more patient compliance but is more forgiving.

Objective: the aim of this work to evaluate the results of acute correction of adolescent Blount's disease using a hinged Ilizarov frame constructed for gradual correction taking the benefits of acute correction and using a hinged frame making it possible to correct any overcorrection or under correction in the clinic.
\end{abstract}

Patients and Methods: It was a prospective study for 23 boys with adolescent Blount disease treated by acute correction using a hinged Ilizarov frame. The average age at presentation was 14 years (range 1117 years), 3 patients (13\%) had bilateral affection. 6 patients had limb length discrepancy.

Results: Average preoperative to postoperative changes of MPTA from 70 (range 60-80) improved to $90^{\circ}$ (range 85-95), Average preoperative to postoperative changes of PPTA from 72 (range 64-80) improved to $80^{\circ}$ (range 78-82), Average preoperative to postoperative changes of MAD from $35 \mathrm{~mm}$ (range $20-50 \mathrm{~mm}$ ) improved to $5 \mathrm{~mm}$ (range $0-10 \mathrm{~mm}$ ). Average preoperative to postoperative changes of TFA from -25 (range -30-20) of internal tibial torsion improved to 5.36 (range $0-10$ ) of external tibial torsion.

Conclusion: Acute correction of adolescent Blount's disease using a hinged two rings Ilizarov frame constructed for gradual correction is a safe technique combining the benefits of acute and gradual correction, allows the surgeon to correct any error at the clinic and decreasing the need for operative fluoroscopy.

Keywords: Blount disease; Hinged ilizarov; Acute correction

Abbreviations: MPTA: Medial Proximal Tibial Angle; PPTA: Posterior Proximal Tibial Angle; MAD: Mechanical Axis Deviation; LLD: Limb Length Discrepancy; TFA: Thigh Foot Angle

\section{Introduction}

Blount's disease or tibia vara is the commonest cause of genu varum in children and adolescents. It is a developmental disease caused by disorder in endochondral ossification of the posteromedial part of the proximal tibial physics resulting in multiplanar deformity of the lower limb with varus, procurvatum and internal tibial torsion [1-3]. Blount disease is classified into early onset (infantile) and late onset. In early onset type the deformity develops before the age of four years. late onset Blount disease is subdivided into a juvenile type (onset at the age of four to ten years) and an adolescent type (onset after the age of ten years). In many cases there is distal femoral varus and a compensatory distal tibial valgus. Limb length discrepancy (LLD) may be present in some cases. This complex deformity can lead to gait abnormalities and premature arthritis of the knee [4-7].

There are many treatment options for Blounts disease including proximal tibial osteotomy, guided growth, and medial plateau elevation Proximal tibial osteotomy remains the gold standard management for late onset cases. External fixators, including monolateral and circular fixators are widely used for deformity correction either acutely or gradually. Gradual correction using distraction osteogenesis can treat limb length discrepancy, however it needs patient compliance. Acute correction has a shorter time to healing and more comfortable to patient [9-13]. This aim of this study is to evaluate the results of acute correction of adolescent Blount's disease combining the benefits of both acute and gradual correction in one treatment modality by acute correction using a hinged frame constructed for gradual correction. 


\section{Patients and Methods}

After approval of the Ethical Committee and a written informed consent from all patients were obtained, and a total of 23 patients with adolescent Blount disease treated by acute correction using a hinged Ilizarov frame were prospectively evaluated between (May 2017-December 2018) this study was done in Department of Orthopedic, Mansoura Health Insurance Hospital. It was a prospective study for 23 boys with adolescent Blount disease treated by acute correction using a hinged Ilizarov frame. The average age at presentation was 14 years (range 11-17 years) , 3 patients (13\%) had bilateral involvement. 6 patients had limb length discrepancy with average $2 \mathrm{~cm}$ (range $1-3 \mathrm{~cm}$ ).

\section{Preoperative planning}

A detailed history taking, clinical examination of the patient and the affected limb was done to exclude the presence of skeletal dysplasia, short stature and leg length discrepancy (Figure). The limb was examined for range of motion, ligamentous laxity, neurovascular status and skin condition. Radiographic examination included long standing X- ray films and CT scanogram to evaluate the mechanical axis and limb length (Figure 1). Short films for the tibia and femur on both sides were also obtained.

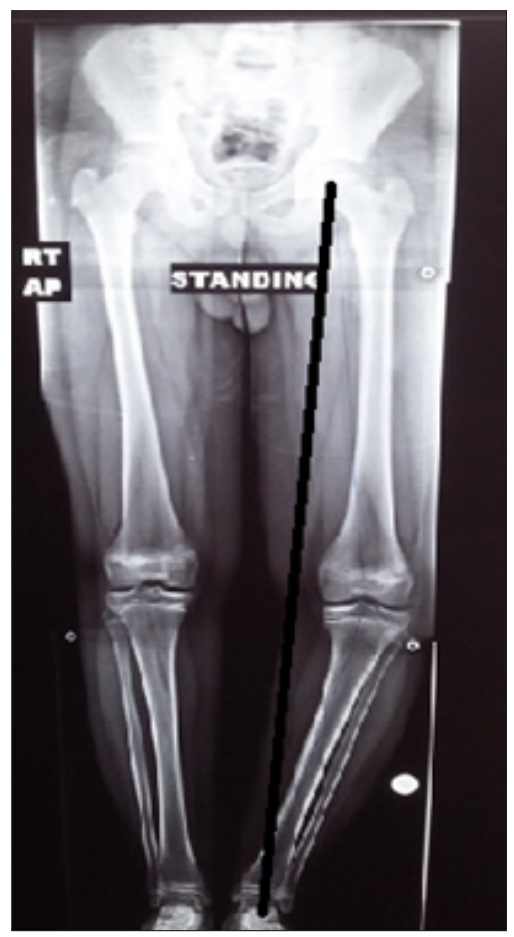

Figure 1: Preoperative long standing $x$ ray film of lower limb of a 14-year-old boy showing left adolescent blounts with medial axis deviation (black line).

\section{Surgical technique}

The operation was performed under general or spinal anesthesia with intravenous antibiotic given one hour preoperative. No tourniquet was used to check vascularity and the patient was put in supine position. The affected limb was sterilized from upper thigh to foot. Fibular osteotomy was the first step done at the junction between middle and lower third. A small incision about $2 \mathrm{~cm}$ was done, incising skin, superficial fascia and deep fascia. Multiple drill holes were done then osteotomy was completed using a thin osteotome.

A simple Ilizarov frame composed of two rings with appropriate size was applied to the leg, with the proximal ring taking the shape of deformity and put at the level of knee joint. The proximal ring was placed parallel to tibial articular surface in all planes. The distal ring was at the level of mid-diaphyseal. One anterior and another posterior hinge were applied to the proximal ring at the level of joint line and to the convex side. The two hinges were fixed by two rods which were attached to the corresponding holes of the distal ring. The proximal ring was fixed to the medial aspect of proximal tibia with 3 or $4-6 \mathrm{~mm}$ schanz screw in two different planes parallel to the joint line. The distal ring was fixed by $4-6 \mathrm{~mm}$ schanzes at two different level and planes through the medial surface of tibia and perpendicular to the shaft. Predrilling was done then manual insertion of schanzes to avoid thermal necrosis to bone. Osteotomy of tibia was done about $3 \mathrm{~cm}$ below tibial tuberosity using multiple drill holes and osteotome. Osteotomy was done through two small incisions one at the shin and the other at the posteromedial cortex of the tibia. One of the hinges was disconnected from proximal ring and external rotation of distal tibia done to assure complete osteotomy. The Hine was fixed back to the same hole and acute correction done using a media distractor fixed in the midsagittal distance between the two hinges. The critical point is to put the hinges at the CORA (joint line) which makes obligatory accurate correction by applying osteotomy rule two [14].

Accurate correction was checked using a diathermy cable from centre of femoral head to the centre of ankle joint passing through the centre of knee joint. After checking accurate deformity correction. The frame was tightened. In very obese patients, a fourth rod was applied at the lateral aspect. In most cases, internal rotation was corrected by medial distraction alone. If there is residual internal rotation, the hinges were moved one or two holes in the proximal ring to externally rotate the shaft. A drain was inserted through the osteotomy wound and closure of skin done, and dressing put. Distal pulse was checked during surgery.

\section{Post-operative care}

Postoperative antibiotics and analgesics were given. patients were discharged from the hospital on the second or third postoperative day. An immediate postoperative $\mathrm{x}$ ray of the knee and tibia was done (Figure 2). Weight bearing as tolerated was allowed and range of movement encouraged. Follow up at the clinic was at second week postoperative then monthly. A long standing $\mathrm{X}$ ray film (Figure 2 ) was done in the first visit to check correction. If there is overcorrection or under correction, the distractor was shortened or lengthened respectively. Patients were teached how to do pin site care daily using normal saline or alcohol. Full weight bearing was usually obtained in the first month. 


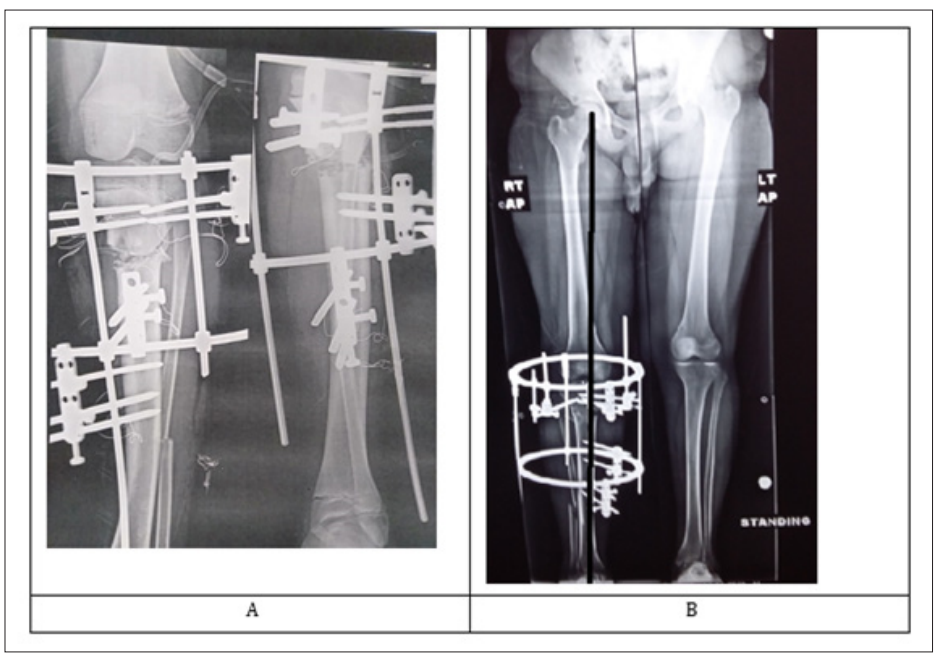

Figure 2: A) Postoperative $x$ ray of left knee and tibia.

B) Long standing $\mathrm{x}$ ray film of another patient showing restoration of normal medial proximal tibia angle and limb alignment.

After radiological healing, the nuts were loosened to be sure of clinical healing and to avoid refracture then frame removed after one week. Two patients had under correction and were adjusted at the first follow up. In patients having residual limb length discrepancy, distraction osteogenesis was started two weeks postoperative one $\mathrm{mm}$ per day till obtaining equal length. In patients with adjustment of the frame postoperative, long X ray film and CT scanogram was repeated. Frame removal was done under sedation. Above knee cast was applied in patients weighting more than $100 \mathrm{~kg}$. X ray film of the knee and tibia was done after frame removal (Figure 3 )The average time of follow up was 9 months (range 5-13).

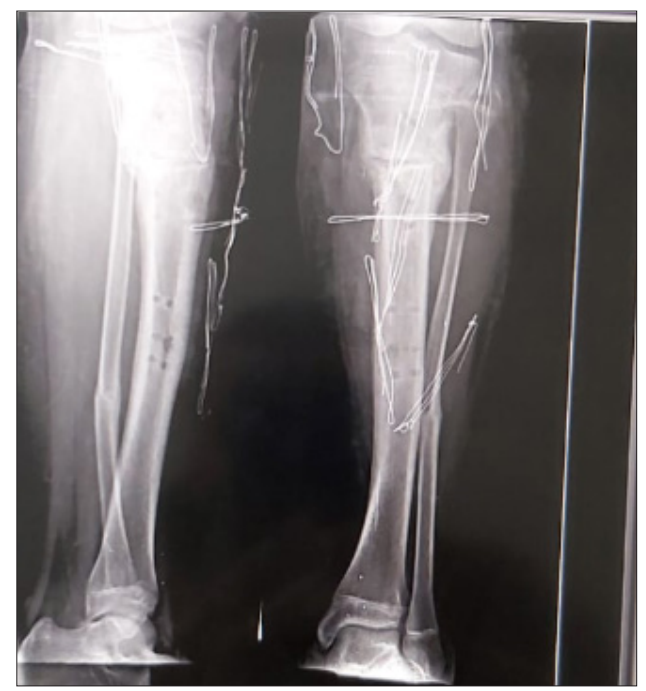

Figure 3: Showing AP and lateral radiographs of knee and leg after frame removal, with good healing and alignment.

\section{Results}

Patients results were assessed both clinically and radiological (Figure 4). Restoration of mechanical axis, knee range of movement, weight bearing, range of motion (ROM) external fixator time and possible complications were assessed. Average preoperative to postoperative changes of Medial proximal tibial angle (MPTA) from $70^{\circ}$ (range 60-80) improved to $90^{\circ}$ (range 85-95). Average preoperative to postoperative changes of Posterior proximal tibial angle (PPTA) from 72 (range 64-80) improved to $80^{\circ}$ (range 78-82). Average preoperative to postoperative changes of Mechanical axis deviation (MAD) from $35 \mathrm{~mm}$ (range $20-50 \mathrm{~mm}$ ) improved to $5 \mathrm{~mm}$ (range $0-10 \mathrm{~mm}$ ). Average preoperative to postoperative changes of Thigh foot angle (TFA) from -25 (range -30-20) of internal tibial torsion improved to $5^{-}$(range $0-10$ ) of external tibial torsion. The average external fixator time was 12 weeks (range 10-14 weeks). 
All osteotomies healed with no re-fracture or loss of correction after fixator removal. Two patients had knee stiffness which responded to physiotherapy. All patients were able to achieve full knee flexion and extension during last follow-up.

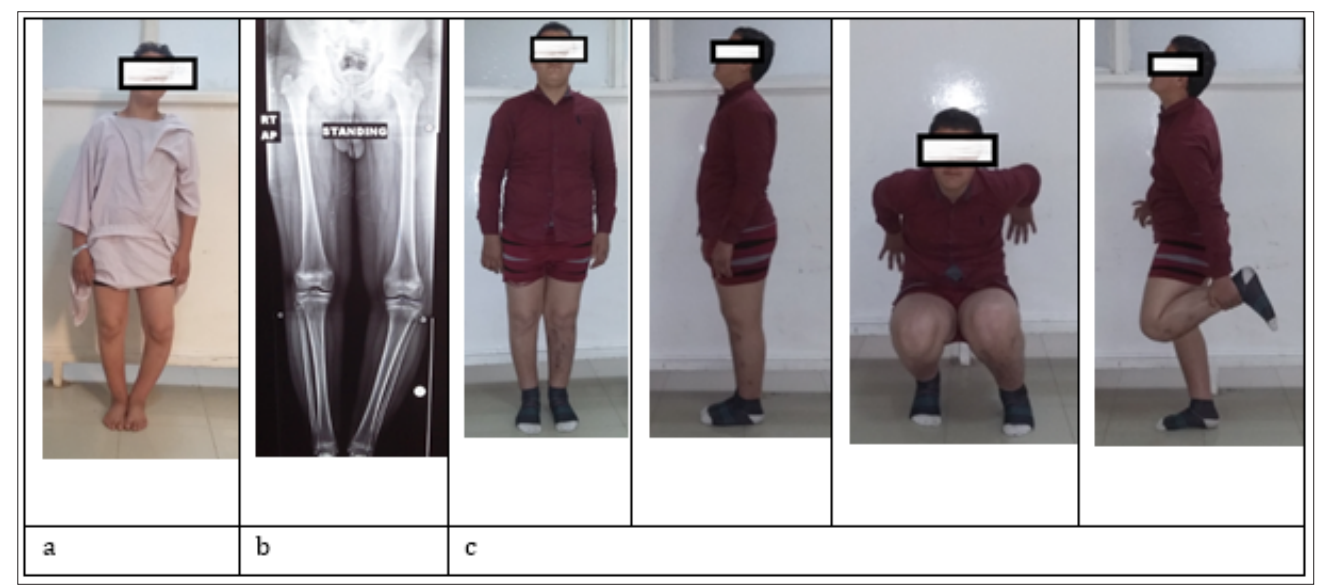

Figure 4: a) clinical radiograph of 14 years old boy with left blount,

b) long x ray film of the same patient,

c) clinical radiographs at the final follow up showing excellent alignment and function.

\section{Complications}

All patients had mild pin site infections, which responded to pin site care and antibiotic treatment. No neurovascular complications or compartment syndrome occurred. No recurrence of deformity occurred.

\section{Conclusion}

We conclude that acute correction of adolescent Blount's disease using a frame constructed for gradual correction is a safe and reliable technique. It combines the benefits of both acute and gradual correction allowing for shorter healing and to correct any operative error at the clinic and reducing the need for intraoperative fluoroscopy.

\section{References}

1. Sabharwal S (2009) Current concepts review: Blount disease. J Bone Joint Surg Am 91(7): 1758-1776.

2. Blount WP (1937) Tibia vara. Osteochondrosis deformans tibiae. J Bone Joint Surg Am 19: 1-29.

3. Sabharwal S, Lee J, Zhao C (2007) Multiplanar deformity analysis of untreated blount disease. J Pediatr Orthop 27(3): 260-265.

4. Amer AR, Khanfour AA (2010) Evaluation of treatment of late onset tibia vara using gradual angulation translation high tibial osteotomy. Acta Orthop Belg 76(3): 360-366.
5. Birch JG (2013) Blount disease. J Am Acad Orthop Surg 21(7): 408-418.

6. Davidson RS (2011) The MAC (multi-axial correcting) monolateral external fixation system (biomet/EBI) technique: An easier way to correct deformity. Oper Tech in Orthop 21(2): 113-124.

7. Gilbody J, Thomas G, Ho K (2009) Acute versus gradual correction of idiopathic tibia vara in children: A systematic review. J Pediatr Orthop 29(2): 110-114.

8. Probe RA (2003) Lower extremity angular malunion: Evaluation and surgical correction. J Am Acad Orthop Surg 11(5): 302-311.

9. Sakurakichi $\mathrm{K}$, Tsuchiya $\mathrm{H}$, Kabata $\mathrm{T}$, Yamashiro $\mathrm{T}$, Watanabe $\mathrm{K}$, et al. (2005) Correction of juxtaarticular deformities in children using the Ilizarov apparatus. J Orthop Sci 10(4): 360-366.

10. Stanitski DF, Dahl M, Louie K, Grayhack J (1997) Management of lateonset tibia vara in the obese patient by using circular external fixation. J Pediatr Orthop 17(5): 691-694.

11. Feldman DS, Madan SS, Koval KJ, Van Bosse HJ, Bazzi J, et al. (2003) Correction of tibia vara with six axis deformity analysis and the Taylor Spatial Frame. J Pediatr Orthop 23(3): 387-391.

12. El Rosasy M, Khanfour A (2014) Late onset tibia vara. Acute correction strategy: proper case selection and a simple external fixation technique. Actaorthop Belg 80(2): 241-250.

13. Feldman DS, Madan SS, Ruchelsman DE, Sala DA, Lehman WB (2006) Accuracy of correction of tibia vara: Acute versus gradual correction. J Pediatr Orthop 26(6): 794-798.

14. Paley D, Herzenberg JE (2002) Principle of deformity correction. $\left(1^{\text {st }}\right.$ edn), Springer-Verlag, Berlin, Germany, pp. 99-145. 